\title{
Four-Dimensional Signal Constellations Based on Binary Frequency-Shift Keying and $M$-ary Amplitude-Phase-Shift Keying
}

\author{
Nodar Ugrelidze1, Sergo Shavgulidze ${ }^{2}$, Juergen Freudenberger ${ }^{3}$ \\ ${ }^{1}$ School of Technology, Caucasus University, Tbilisi, Georgia \\ ${ }^{2}$ Faculty of Power Engineering and Telecommunications, Georgian Technical University, Tbilisi, Georgia \\ ${ }^{3}$ Institute for System Dynamics, HTWG Konstanz, University of Applied Sciences, Konstanz, Germany \\ Email: toban555@gmail.com, s.shavgulidze@gtu.ge,jfreuden@htwg-konstanz.de
}

How to cite this paper: Ugrelidze, N., Shavgulidze, S. and Freudenberger, J. (2020) Four-Dimensional Signal Constellations Based on Binary Frequency-Shift Keying and $M$-ary Amplitude-Phase-Shift Keying. Journal of Computer and Communications, $\mathbf{8}$, 170-180.

https://doi.org/10.4236/jcc.2020.812016

Received: November 25, 2020

Accepted: December 22, 2020

Published: December 25, 2020

Copyright $\odot 2020$ by author(s) and Scientific Research Publishing Inc. This work is licensed under the Creative Commons Attribution International License (CC BY 4.0).

http://creativecommons.org/licenses/by/4.0/

\begin{abstract}
In this article, we give the construction of new four-dimensional signal constellations in the Euclidean space, which represent a certain combination of binary frequency-shift keying (BFSK) and $M$-ary amplitude-phase-shift keying (MAPSK). Description of such signals and the formulas for calculating the minimum squared Euclidean distance are presented. We have developed an analytic building method for even and odd values of $M$. Hence, no computer search and no heuristic methods are required. The new optimized BFSK-MAPSK ( $M=5,6, \cdots, 16)$ signal constructions are built for the values of modulation indexes $h=0.1,0.15, \cdots, 0.5$ and their parameters are given. The results of computer simulations are also provided. Based on the obtained results we can conclude, that BFSK-MAPSK systems outperform similar fourdimensional systems both in terms of minimum squared Euclidean distance and simulated symbol error rate.
\end{abstract}

\section{Keywords}

Signal Constellation, Minimum Squared Euclidean Distance, Modulation Index, Signal Phase, Simulation, Symbol Error Rate

\section{Introduction}

To date, there has been a significant increase in interest in digitally modulated signals, mainly due to the widespread use of multi-input and multi-output (MIMO) systems. Of the well-known effective signal systems, multi-dimensional signals have a better performance of error rate (symbol error rate-SER or bit error rate-BER) compared to conventional two-dimensional signals [1] [2] [3]. 
It is well known that multidimensional signals can be formed using time or frequency (or both) spaces [2] [3] [4] [5]. Concerning SER characteristics good results are obtained for four-dimensional (4D) signals, which represent certain combinations of binary frequency shift keying (BFSK) and $M$-ary phase-shift keying (MPSK) modulations (BFSK-MPSK signals). Initially, several such signals were presented in combination with channel coding schemes [4] [5], and later, as the components of Spatial Modulation (SM) systems [6]. In both cases, the considered signals gave a remarkable performance.

Numerous new 4D BFSK-MPSK signal characteristics are listed in [7], where their advantage (according to SER) is shown compared to the best $M$-ary amplitude-phase shift keying (MAPSK) signals discussed in [1] [8]. The constellations with usual size ( $M=2^{i}$, where $i$ is a natural number), as well as atypical size $\left(M \neq 2^{i}\right)$ are presented in [6], whose use has been particularly convenient and fruitful in MIMO SM systems.

To further improve the signal distance and, accordingly, SER characteristics, in this article we will discuss $4 \mathrm{D}$ constructions of hybrid type constellations of size $M$, which will be built based on a combination of BFSK and MAPSK modulations (the so-called BFSK-MAPSK signals). The main novelty of this paper is the presentation of a general regular method of constructing such $4 \mathrm{D}$ constellations that uses an analysis line different from [7] and for particular cases leads to the optimal construction of [7]. In the new scheme, MAPSK modulation uses two-level circular signal constellations with energy levels-high $\left(E_{H}\right)$ and low $\left(E_{L}\right)$. We consider $E_{H}=k \cdot E_{L}$, where $k>1$ and the signals with the same energy levels are transmitted at the same frequencies. Using the proposed method, for modulation indexes $h \leq 0.5$ and alphabet sizes $5 \leq M \leq 16$, we construct new BFSK-MAPSK signal constellations and present the parameters of the best of them in the form of a table. Furthermore, we illustrate their advantages over systems from [4] [5] [7] concerning the SER performance using computer simulations for the additive white Gaussian noise channel.

MIMO SM techniques appear as competitive candidates for next-generation (5G and beyond) wireless networks due to the attractive advantages they offer in terms of spectral and energy efficiency as well as hardware simplicity. We think that the presented signals can be effectively used in various multiantenna SM realizations developed by us [9] [10]. It should also be noted, that new four-dimensional signals can operate in the similar coded modulation schemes which are described in [2] [3] [4] [5].

The rest of this paper is organized as follows: Section 2 describes BFSK-MAPSK signals, their parameters, and distance characteristics. Section 3 presents signal construction methodology and uses it to calculate the best parameters of the signal system. The results of simulation and comparative analysis are shown in Section 4 whereas the conclusions are figured out in Section 5.

\section{The Description and Parameters of BFSK-MAPSK}

In the case of the BFSK-MAPSK signal (or BFSK-MAPSK signal constellation $C$ ) 
two different frequencies $\omega_{c}+\pi h / T_{s} \mathrm{rad} / \mathrm{s}$ and $\omega_{c}-\pi h / T_{s} \mathrm{rad} / \mathrm{s}\left(T_{s}\right.$ is signal duration) are used to transmit $M 1$-ary and $M 2$-ary PSK signals (namely, M1PSK and M2PSK), respectively. Accordingly, we have two sub-constellations, $C 1$ and $C 2, C=C 1 \cup C 2$. Besides, $M$-ary $(M=M 1+M 2)$ signal configuration is such that $M 1=M 2$ when $M$ is even and $M 2=M 1-1$ when $M$ is odd. The energy of each signal in the $C 1$ sub-constellation is $E_{H}$, and the energy of each signal in $C 2$ sub-constellation is $E_{L}$. The average signal energy in constellation $C$ is

$\left(M 1 \cdot E_{H}+M 2 \cdot E_{L}\right) / M=1$.

For the considered cases we have

$$
\begin{cases}E_{L}=2 /(1+k), E_{H}=2 k /(1+k), & \text { if } M \text { is even; } \\ E_{L}=2 /\left(\frac{M+1}{M} \cdot k+\frac{M-1}{M}\right), E_{H}=2 k /\left(\frac{M+1}{M} \cdot k+\frac{M-1}{M}\right), & \text { if } M \text { is odd. }\end{cases}
$$

From (1) follows that it is sufficient to know the value of $k$ to determine the energies of the $M$-ary signals in the process of their optimization. As an example, Figure 1 shows the BFSK-8APSK constellation, where the signal vector norm is $\sqrt{E_{H}}$ in the $C 1$ sub-constellation and $\sqrt{E_{L}}$ in the $C 2$ sub-constellation, respectively.

Analytically, the BFSK-MAPSK signal can be defined as follows:

$$
s_{\tau}(t)=\sqrt{\frac{2 E_{s}}{T_{s}}} \cos \left[\left(\omega_{c} \pm \frac{\pi h}{T_{s}}\right) t+\varphi_{\tau}\right], 0 \leq t \leq T_{s},
$$

where $E_{s}$ is the signal energy, $E_{s} \in\left\{E_{H}, E_{L}\right\} ; \varphi_{\tau}$ is the phase of the signal $s_{\tau}(t),(\tau \in\{1,2, \cdots, M\})$. It is important to note that the following conditions will always be satisfied: $E_{s}=E_{H}$, when $\tau$ is odd, and $E_{s}=E_{L}$ when $\tau$ is even.

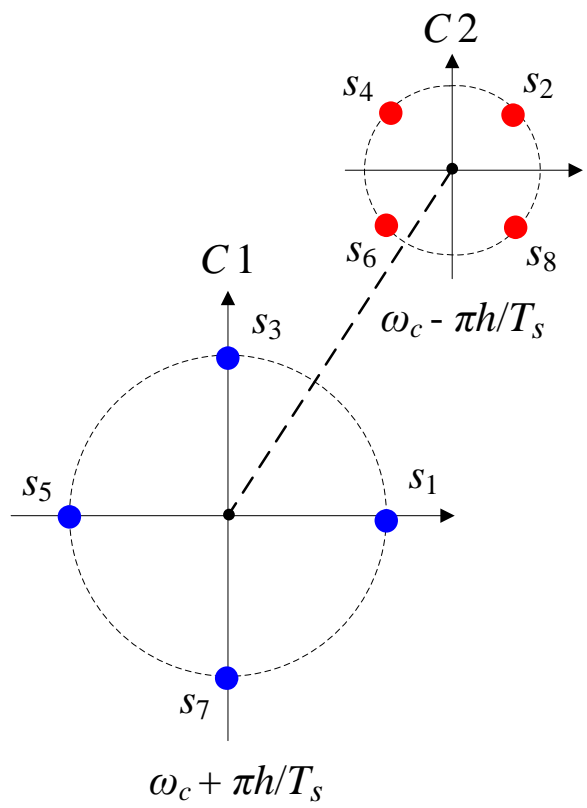

Figure 1. Presentation of 4D BFSK-8APSK constellation. 
In [7] based on the Gram-Schmidt orthonormalization procedure for BFSKMPSK signals we constructed the basic vectors of $4 \mathrm{D}$ space. Similarly, for BFSKMAPSK signal coordinates we get:

$S_{\tau}= \begin{cases}\sqrt{E_{H}}\left(\cos \varphi_{\tau}, \sin \varphi_{\tau}, 0,0\right), & \text { for } C 1 ; \\ \sqrt{E_{L}}\left(K_{1} \cos \varphi_{\tau}+K_{2} \sin \varphi_{\tau}, K_{1} \sin \varphi_{\tau}-K_{2} \cos \varphi_{\tau}, \sqrt{D} \cos \varphi_{\tau}, \sqrt{D} \sin \varphi_{\tau}\right), & \text { for } C 2,\end{cases}$

where $K_{1}=(\sin 2 \pi h) / 2 \pi h, K_{2}=(1-\cos 2 \pi h) / 2 \pi h, D=1-K_{1}^{2}-K_{2}^{2}$.

It is obvious, that for $C 1$ sub-constellation $\tau$ is odd and for $C 2$ sub-constellation $\tau$ is even.

Later on, we will use the general formula for calculation of the squared Euclidean distance between $s_{i}$ and $s_{j}$ signals [1]:

$$
d^{2}\left(s_{i}, s_{j}\right)=\int_{0}^{T_{s}}\left[s_{i}(t)-s_{j}(t)\right]^{2} \mathrm{~d} t .
$$

Then taking (2) and (4) into account, we obtain the following formulas for the squared Euclidean in sub-constellations $C 1$ and $C 2$ :

$$
\begin{aligned}
& d^{2}(C 1)=2 E_{H}[1-\cos (\Delta \varphi(C 1))], \\
& d^{2}(C 2)=2 E_{L}[1-\cos (\Delta \varphi(C 2))],
\end{aligned}
$$

where $\Delta \varphi(C 1)$ and $\Delta \varphi(C 2)$ are the phase differences between the corresponding signals for each sub-constellation.

The squared Euclidean distance between the $i$-th signal $s_{i}(i \in\{1,3, \cdots\})$ of $C 1$ sub-constellation and $j$-th signal $s_{j}(j \in\{2,4, \cdots\})$ of $C 2$ sub-constellation can be calculated as follows:

$$
d^{2}\left(s_{i}, s_{j}\right)=E_{H}+E_{L}-2 \operatorname{sinc}(\pi h) \cdot \sqrt{E_{H} E_{L}} \cdot \cos \left(\varphi_{i}-\varphi_{j}^{s}\right),
$$

where $\operatorname{sinc}(\pi h)=\sin (\pi h) /(\pi h), \varphi_{i}$ is initial phase of $s_{i}$ signal, $\varphi_{j}^{s}=\varphi_{j}-\pi h$ and $\varphi_{j}$ is the initial phase of $s_{j}$ signal.

\section{The Construction of BFSK-MAPSK Signals}

First, consider the case when $M$ is even. Suppose, that we have the basic constellation $C_{s}$ with elements $s_{1}, s_{2}, \cdots, s_{M}$ with even $M$ and the initial phases $\varphi_{1}, \varphi_{2}, \cdots, \varphi_{M}$, respectively. Furthermore, for any $m \in\{1,2, \cdots,(M-1)\}$, $\varphi_{m+1}>\varphi_{m}$ and $\varphi_{m+1}-\varphi_{m}=\phi_{e}=2 \pi / M$.

Let us partition $C_{s}$ into the sub-constellations $C_{s} 1$ and $C_{s} 2$ so that $\left\{s_{i}\right\}_{i=1,3, \ldots} \in C_{s} 1$ and $\left\{s_{j}\right\}_{j=2,4, \ldots} \in C_{s} 2$. The corresponding values of the signal phases are $\left\{\varphi_{i}\right\}_{i=1,3, \cdots}$ and $\left\{\varphi_{j}\right\}_{j=2,4, \ldots}$. For further use, we denote $\left\{\varphi_{j}\right\}$ by $\left\{\varphi_{j}^{s}\right\}$. For example, the phase diagrams of the basic constellation $C_{s}$ as well as the sub-constellations $C_{s} 1$ and $C_{s} 2$ for $M=8$ are presented in Figure 2:

We say that $C_{s} 1$ and $C_{s} 2$ sub-constellations have signal energies $E_{H}$ and $E_{L}$, respectively. As it was noted in the introduction part, we can assume that $E_{H}>E_{L}$ and $E_{H}=k \cdot E_{L}, k>1$.

We can define the minimum values of the squared Euclidean distances for signals of $C_{s} 1$ and $C_{s} 2$ sub-constellations as follows: 

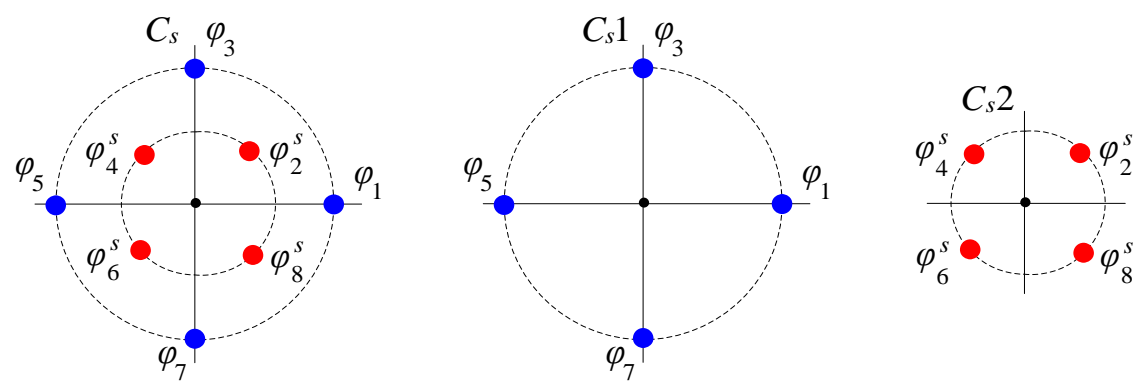

Figure 2. Example of a basic constellation and its two sub-constellations for $M=8$.

$$
\begin{aligned}
& d_{\text {min }}^{2}\left(C_{s} 1\right)=2 E_{H}(1-\cos (4 \pi / M)), \\
& d_{\min }^{2}\left(C_{s} 2\right)=2 E_{L}(1-\cos (4 \pi / M)) .
\end{aligned}
$$

Taking (7) into account, we define the minimum value of the squared Euclidean distance between the signals from the sub-constellation $C_{s} 1$ and $C_{s} 2$ as follows:

$$
d_{\min }^{2}\left(C_{s} 1, C_{s} 2\right)=E_{L}(k-2 \operatorname{sinc}(\pi h) \cdot \sqrt{k} \cdot \cos (2 \pi / M)+1) .
$$

Given that $d_{\min }^{2}\left(C_{s} 1\right)>d_{\min }^{2}\left(C_{s} 2\right)$, the value of minimum squared Euclidean distance for $C_{s}$ constellation will be:

$$
d_{\min }^{2}\left(C_{s}\right)=\min \left[d_{\min }^{2}\left(C_{s} 2\right), d_{\min }^{2}\left(C_{s} 1, C_{s} 2\right)\right] .
$$

It is not difficult to see that in (11) $d_{\min }^{2}\left(C_{s} 2\right)$ is a monotonically decreasing function with respect to $k$ for a given $M$, while $d_{\min }^{2}\left(C_{s} 1, C_{s} 2\right)$ is a monotonically increasing function with respect to a given $M$ and $h$. The intersection point of these functions determines the optimal value of $k$. Undoubtedly, this point will exist if the condition is fulfilled:

$$
\left[d_{\min }^{2}\left(C_{s} 2\right) \geq d_{\min }^{2}\left(C_{s} 1, C_{s} 2\right)\right]_{k=1} .
$$

Let us say, that $d_{\min }^{2}\left(C_{s} 2\right)=d_{\min }^{2}\left(C_{s} 1, C_{s} 2\right)$. Then the following equality is true:

$$
k-2 \operatorname{sinc}(\pi h) \cdot \cos (2 \pi / M) \cdot \sqrt{k}+2 \cos (4 \pi / M)-1=0 .
$$

From (13) we get the solution for $k \geq 1$ :

$k=\left[\operatorname{sinc}(\pi h) \cdot \cos (2 \pi / M) \pm \sqrt{(\operatorname{sinc}(\pi h) \cdot \cos (2 \pi / M))^{2}-(2 \cos (4 \pi / M)-1)}\right]^{2}$

If we use the already known values of $\phi_{e}$ and $k$ we can fully determine the optimal $C_{s}$ basic constellation (with respect to the $\max d_{\min }^{2}\left(C_{s}\right)$ ) and at this time $d_{\min }^{2}\left(C_{s}\right)=d_{\min }^{2}\left(C_{s} 2\right)$.

Considering (7), the procedure for obtaining optimized BFSK-MAPSK constellations $C(C=C 1 \bigcup C 2)$ will be as follows: sub-constellation $C 1$ must coincide with $C_{s} 1$ and the sub-constellation $C 2$ is obtained by adding $\pi h$ (modulo $2 \pi)$ to the values of the phases of the $C_{s} 2$ sub-constellation. It is clear that for the constellation $C$ the following equation holds: 


$$
d_{\text {min }}^{2}(C)=2 E_{L}(1-\cos (4 \pi / M)) \text {. }
$$

Next, consider the case when $M$ is odd. As in the previous case, we have the basic constellation $C_{s}$ with an odd number of elements $s_{1}, s_{2}, \cdots, s_{M}$ with initial phases values $\varphi_{1}, \varphi_{2}, \cdots, \varphi_{M}$, respectively. However, for any $m \in\{1,2, \cdots,(M-1)\}, \varphi_{m+1}>\varphi_{m}$ and

$$
\varphi_{m+1}-\varphi_{m}=\phi_{o}=\varphi_{M} /(M-1) .
$$

Just like to Figure 2, for $M=7$ we have the constellation presented in Figure 3.

Similar to the case of even $M$, for odd $M$ the minimum squared Euclidean distance of the basic constellation $C_{s}$ is

$$
d_{\min }^{2}\left(C_{s}\right)=\min \left[d_{\min }^{2}\left(C_{s} 2\right), d_{\min }^{2}\left(C_{s} 1, C_{s} 2\right), d^{2}\left(s_{1}, s_{M}\right)\right],
$$

where

$$
\begin{gathered}
d_{\min }^{2}\left(C_{s} 2\right)=2 E_{L}\left(1-\cos \left(2 \phi_{o}\right)\right), \\
d_{\min }^{2}\left(C_{s} 1, C_{s} 2\right)=E_{L}\left(k-2 \operatorname{sinc}(\pi h) \cdot \sqrt{k} \cdot \cos \left(\phi_{o}\right)+1\right), \\
d^{2}\left(s_{1}, s_{M}\right)=2 E_{H}\left(1-\cos \left((M-1) \phi_{o}\right)\right) .
\end{gathered}
$$

It is easy to show that likewise to the case of even $M$, the condition of existence of optimal basic $C_{s}$ constellation is $d_{\min }^{2}\left(C_{s} 2\right)=d_{\min }^{2}\left(C_{s} 1, C_{s} 2\right)$. However, this is a necessary but insufficient condition, as there exists a possibility that the square of the Euclidean distance between the first and last signals of the basic constellation will be $d^{2}\left(s_{1}, s_{M}\right)<d_{\min }^{2}\left(C_{s} 2\right)$ and $d^{2}\left(s_{1}, s_{M}\right)<d_{\min }^{2}\left(C_{s} 1, C_{s} 2\right)$.

Therefore, the necessary and sufficient condition for the optimality of $C_{s}$ will be:

$$
d_{\text {min }}^{2}\left(C_{s} 2\right)=d_{\text {min }}^{2}\left(C_{s} 1, C_{s} 2\right)=d^{2}\left(s_{1}, s_{M}\right) .
$$

Given that $d_{\min }^{2}\left(C_{s} 2\right)=d^{2}\left(s_{1}, s_{M}\right)$ we will get the formula

$$
k=\frac{\left(\sin \left(\phi_{o}\right)\right)^{2}}{\left(\sin \left((M-1) \phi_{o} / 2\right)\right)^{2}},
$$

where $\phi_{o}$ is defined by (16). If we take into consideration that $d_{\text {min }}^{2}\left(C_{s} 2\right)=d_{\text {min }}^{2}\left(C_{s} 1, C_{s} 2\right)$ then the following equation is true
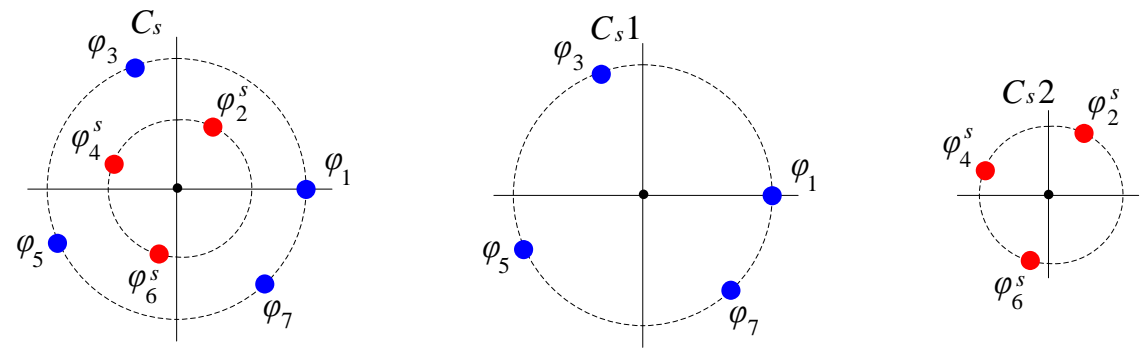

Figure 3. Example of a basic constellation and its two sub-constellations for $M=7$. 


$$
\begin{aligned}
& \left(\sin \left(\varphi_{M} /(M-1)\right)\right)^{2}-\operatorname{sinc}(\pi h) \cdot \sin \left(2 \varphi_{M} /(M-1)\right) \cdot \sin \left(\varphi_{M} / 2\right) \\
& +\left(\sin \left(\varphi_{M} / 2\right)\right)^{2} \cdot\left(1-4\left(\sin \left(\varphi_{M} /(M-1)\right)\right)^{2}\right)=0,
\end{aligned}
$$

from which we can determine $\varphi_{M}$.

The Equation (23) can have many solutions and we choose from the $\left\{\varphi_{M i}\right\}_{i>1}$ the maximum real solution less than $2 \pi$, which ensures the maximization of the $d_{\text {min }}^{2}\left(C_{s} 2\right)$ values.

Finally, according to the given $k$ and $\phi_{o}$ we get the basic constellation $C_{s}$, from which we can obtain the optimized BFSK-MAPSK signal constellation $C$ by a procedure, similar to the case of the even $M$, that is with the addition (modulo $2 \pi$ ) of the values of $\pi h$ to the phases to the $C_{s} 2$ sub-constellation. Finally, it follows from the above discussions, that for odd $M$ we have

$$
d_{\text {min }}^{2}(C)=2 E_{L}\left(1-\cos \left(2 \phi_{o}\right)\right) \text {. }
$$

It should be noted, that without loss of generality, we can assume that $\varphi_{1}=0$ for both even and odd values of $M$.

It follows from [7] that minimum squared Euclidean distance for BFSK-MPSK signals can be calculated as follows:

$$
d_{\min (\text { BFSK-MPSK })}^{2}=2(1-\operatorname{sinc}(\pi h) \cdot \cos (2 \pi / M)) .
$$

Taking into consideration that MAPSK signal systems have better minimum squared Euclidean distance values compared to MPSK, it can be presumed that for certain values of $h$ and $M$, BFSK-MAPSK would have better values of $d_{\min }^{2}$ compared to BFSK-MPSK, which leads to the suggestion that BFSK-MAPSK should ensure better SER performance compared to BFSK-MPSK. Then the question naturally arises as to what values of $M$ and $h$ this case could take place. Let us show the principle of solving this problem for even values of $M$.

If for BFSK-MAPSK signals the right-hand side of the condition (25) is valid then the minimum squared Euclidean distance is equal to

$$
d_{\min (\text { BFSK-MAPSK })}^{2}=2 E_{L}(1-\cos (4 \pi / M))
$$

and for interesting us case we have

$$
2 E_{L}(1-\cos (4 \pi / M))>2(1-\operatorname{sinc}(\pi h) \cdot \cos (2 \pi / M)) .
$$

Afterwards, taking into account that $E_{L}<1$, we obtain the formula

$$
\operatorname{sinc}(\pi h)>\cos (4 \pi / M) / \cos (2 \pi / M)
$$

and this is a condition under which for given $h$ and $M$, the inequality $d_{\min (\text { BFSK-MAPSK) }}^{2}>d_{\min (\text { BFSK-MPSK) }}^{2}$ holds.

Clearly, with the same approach, a similar formula can be obtained for odd values of $M$.

Table 1 lists some of the BFSK-MAPSK system parameters that we have identified and selected using the methods and procedures described above. Here the values of $\phi_{o}$ and $\phi_{e}$ are given in degrees. 
Table 1. Parameters of BFSK-MAPSK systems.

\begin{tabular}{|c|c|c|c|c|c|c|c|c|c|c|c|c|}
\hline \multirow{2}{*}{$h$} & \multicolumn{2}{|c|}{$M=5$} & \multicolumn{2}{|c|}{$M=6$} & \multicolumn{2}{|c|}{$M=7$} & \multicolumn{2}{|c|}{$M=8$} & \multicolumn{2}{|c|}{$M=9$} & \multicolumn{2}{|c|}{$M=10$} \\
\hline & $\phi_{o}$ & $d_{\min }^{2}$ & $\phi_{e}$ & $d_{\min }^{2}$ & $\phi_{o}$ & $d_{\min }^{2}$ & $\phi_{e}$ & $d_{\min }^{2}$ & $\phi_{o}$ & $d_{\min }^{2}$ & $\phi_{e}$ & $d_{\min }^{2}$ \\
\hline 0.10 & 74.96 & 1.4193 & 60.00 & 1.2105 & 52.11 & 0.9443 & 45.00 & 0.8580 & 39.95 & 0.6983 & 36.00 & 0.6500 \\
\hline 0.15 & 74.92 & 1.4258 & 60.00 & 1.2237 & 52.04 & 0.9574 & 45.00 & 0.8740 & 39.88 & 0.7129 & 36.00 & 0.6664 \\
\hline 0.20 & 74.86 & 1.4348 & 60.00 & 1.2420 & 51.94 & 0.9757 & 45.00 & 0.8966 & 39.78 & 0.7338 & 36.00 & 0.6899 \\
\hline 0.25 & 74.77 & 1.4465 & 60.00 & 1.2654 & 51.81 & 1.0000 & 45.00 & 0.9259 & 39.64 & 0.7613 & 36.00 & 0.7208 \\
\hline 0.30 & 74.68 & 1.4606 & 60.00 & 1.2939 & 51.65 & 1.0290 & 45.00 & 0.9623 & 39.48 & 0.7959 & 36.00 & 0.7598 \\
\hline 0.35 & - & - & 60.00 & 1.3273 & 51.46 & 1.0642 & 45.00 & 1.0057 & 39.27 & 0.8381 & 36.00 & 0.8075 \\
\hline 0.40 & - & - & 60.00 & 1.3654 & 51.24 & 1.1053 & 45.00 & 1.0563 & 39.02 & 0.8886 & 36.00 & 0.8645 \\
\hline 0.45 & - & - & 60.00 & 1.4079 & 50.98 & 1.1523 & 45.00 & 1.1142 & 38.72 & 0.9480 & 36.00 & 0.9314 \\
\hline 0.50 & - & - & 60.00 & 1.4544 & 50.69 & 1.2053 & 45.00 & 1.1790 & 38.36 & 1.0172 & 36.00 & 1.0083 \\
\hline \multirow{2}{*}{$h$} & \multicolumn{2}{|c|}{$M=11$} & \multicolumn{2}{|c|}{$M=12$} & \multicolumn{2}{|c|}{$M=13$} & \multicolumn{2}{|c|}{$M=14$} & \multicolumn{2}{|c|}{$M=15$} & \multicolumn{2}{|c|}{$M=16$} \\
\hline & $\phi_{o}$ & $d_{\min }^{2}$ & $\phi_{e}$ & $d_{\min }^{2}$ & $\phi_{o}$ & $d_{\min }^{2}$ & $\phi_{e}$ & $d_{\min }^{2}$ & $\phi_{o}$ & $d_{\min }^{2}$ & $\phi_{e}$ & $d_{\min }^{2}$ \\
\hline 0.10 & 32.42 & 0.5429 & 30.00 & 0.5125 & 27.31 & 0.4360 & 25.71 & 0.4155 & 23.61 & 0.3585 & 22.50 & 0.3442 \\
\hline 0.15 & 32.36 & 0.5577 & 30.00 & 0.5285 & 27.25 & 0.4503 & 25.71 & 0.4309 & 23.55 & 0.3723 & 22.50 & 0.3589 \\
\hline 0.20 & 32.26 & 0.5790 & 30.00 & 0.5517 & 27.16 & 0.4714 & 25.71 & 0.4535 & 23.46 & 0.3929 & 22.50 & 0.3808 \\
\hline 0.25 & 32.13 & 0.6075 & 30.00 & 0.5828 & 27.03 & 0.5001 & 25.71 & 0.4844 & 23.34 & 0.4217 & 22.50 & 0.4116 \\
\hline 0.30 & 31.97 & 0.6442 & 30.00 & 0.6230 & 26.86 & 0.5381 & 25.71 & 0.5255 & 23.16 & 0.4612 & 22.50 & 0.4543 \\
\hline 0.35 & 31.75 & 0.6902 & 30.00 & 0.6734 & 26.63 & 0.5875 & 25.71 & 0.5793 & 22.89 & 0.5166 & 22.50 & 0.5140 \\
\hline 0.40 & 31.48 & 0.7470 & 30.00 & 0.7357 & 26.31 & 0.6524 & 25.71 & 0.6493 & - & - & - & - \\
\hline 0.45 & 31.12 & 0.8168 & 30.00 & 0.8116 & - & - & - & - & - & - & - & - \\
\hline 0.50 & 30.64 & 0.9026 & 30.00 & 0.9026 & - & - & - & - & - & - & - & - \\
\hline
\end{tabular}

Concerning $d_{\min }^{2}$, all signals in Table 1 are better than the BFSK-MPSK systems presented in [4] [5] [7], especially for small $h(h \leq 0.5)$ and high $M$, which is important for better bandwidth and spectral efficiency. The latter is defined as $S_{E}=\log _{2}(M)$ and $S_{E}=2.322,2.585, \cdots, 4$ in our variants. It is also obvious, that the presented new systems have better $d_{\min }^{2}$ values compared with optimal $M$-ary APSK [8] and $M$-ary PSK [1] signals.

We observe from the performed calculations that the system is optimal if $k \approx 1$ (or $k=1$ ), which means that we have constructed the optimal BFSK-MPSK constellation, where the parameter $k$ is the signal energy ratio of the two energy levels introduced in Section 1, i.e. $k=E_{H} / E_{L}$. Therefore, the optimal BFSK-MAPSK system construction method presented in this paper is a general one, which can be used to obtain the optimal signal system of both types-BFSK-MAPSK or BFSK-MPSK [7]. We note that the method used in [7] allows us to build only BFSK-MPSK.

\section{Simulation Results}

To investigate the effectiveness of the new signals more deeply, the computer 


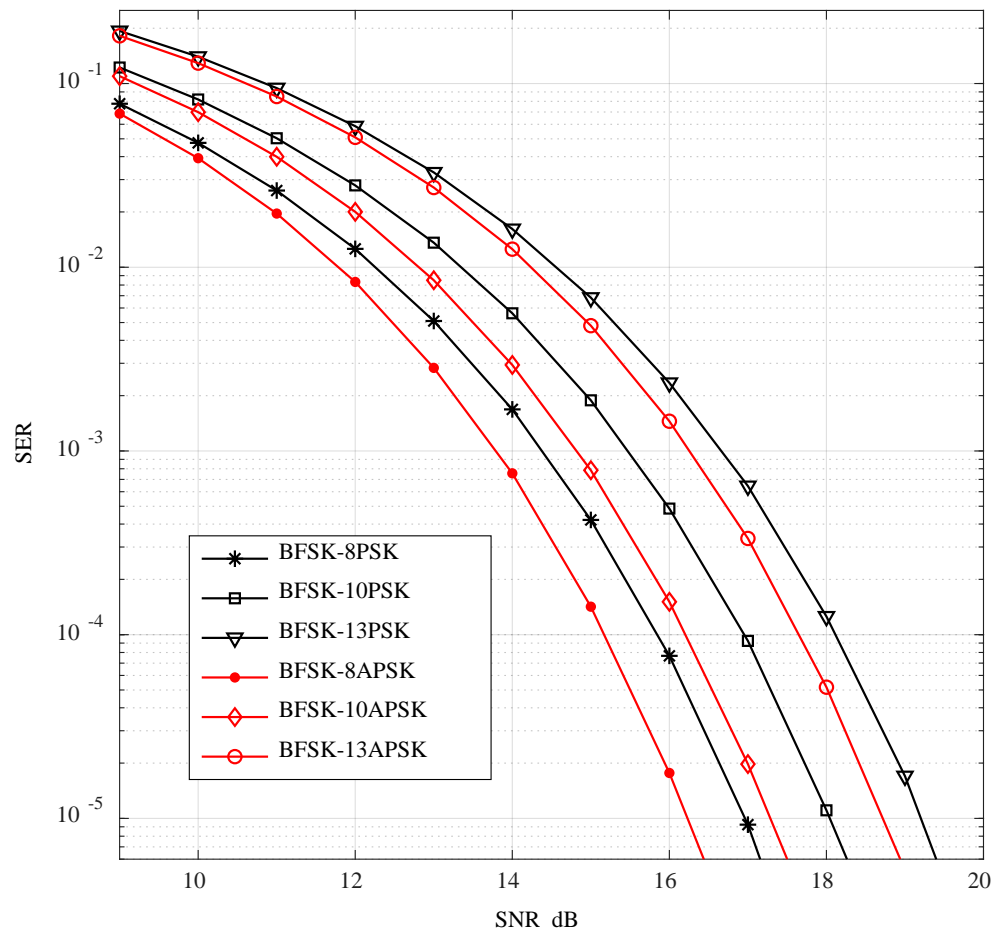

Figure 4. SER characteristics of 4D signals.

simulations of BFSK-MAPSK signals and BFSK-MPSK signals from [7] were conducted in the additive white Gaussian noise channels. The transmission of 50,000,000 $M$-ary symbols was considered. The results are shown in Figure 4, where SNR is the average signal-to-noise ratio per transmitted $M$-ary symbol. For all cases, we used $h=0.3$. The reception of the signals was carried out in Euclidean space by maximum-likelihood detection. The simulation results show that for SER $=10^{-5}$ the energy gain of BFSK-MAPSK signals compared to BFSKMPSK signals are around $0.73 \mathrm{~dB}, 0.76 \mathrm{~dB}$, and $0.48 \mathrm{~dB}$ for $M=8, M=10$ and $M$ $=13$, respectively, which once again indicates the advantage of BFSK-MAPSK over BFSK-MPSK systems. During simulation we used the following values of signal energies and phases (in degrees):

1) BFSK-MPSK: $E_{s}=1, \varphi=\left[\begin{array}{l}099099180189270279\end{array}\right]$ for $M=8 ; E_{s}=1, \varphi=$ [0 187290144162216234288 306] for $M=10 ; E_{s}=1, \varphi=\left[\begin{array}{l}0 \\ 0\end{array} 8.674153 .3481\right.$ $\begin{array}{llllllll}134.0222 & 106.6962 & 187.3703 & 160.0444 & 240.7184 & 213.3925 & 294.0665 & 266.7406\end{array}$ $347.4146320 .0887]$ for $M=13$.

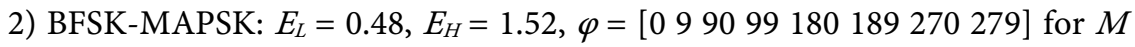
$=8 ; E_{L}=0.55, E_{H}=1.45, \varphi=[0187290144162216234288306]$ for $M=10 ; E_{L}$

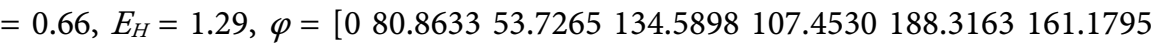
$242.0428214 .9060295 .7693268 .6325349 .4958322 .3590]$ for $M=13$.

\section{Conclusion}

In this article, we have introduced a new signal BFSK-MAPSK constellation consisting of sub-constellations with a different energy. We have presented their 
description and formulas to calculate the distance characteristics. Based on these results we developed the optimized BFSK-MAPSK signaling systems with the help of regular non-computer search and not heuristic methods for both odd and even constellation sizes of $M$ and any modulation index $h$. We have described the procedure that allows us to build better systems compared to BFSK-MPSK in the case of a given $M$ and $h$. We have described the procedure that allows to build efficient BFSK-MAPSK systems and presented the new system parameters with the best $d_{\min }^{2}$ in the form of a table. We also carried out computer simulations. The proposed BFSK-MAPSK systems outperform similar four-dimensional systems in terms of minimum squared Euclidean distance and simulated symbol error rate.

\section{Acknowledgements}

The work was funded by the Shota Rustaveli National Science Foundation (SRNSF FR-19-105) and the Deutsche Forschungsgemeinschaft (DFG, German Research Foundation FR 2673/6-1).

\section{Conflicts of Interest}

The authors declare no conflicts of interest regarding the publication of this paper.

\section{References}

[1] Proakis, J.G. and Salehi, M. (2008) Digital Communications. 5th Edition, McGraw Inc., New York.

[2] Forney, G.D. and Wei, L.F. (1989) Multidimensional Constellations-Part I. Introduction, Figures of Merit, and Generalized Cross Constellations. IEEE Journal on Selected Areas in Communications, 7, 877-892. https://doi.org/10.1109/49.29611

[3] Freudenberger, J. and Shavgulidze, S. (2015) New Four-Dimensional Signal Constellations From Lipschitz Integers for Transmission Over the Gaussian Channel. IEEE Transactions on Communications, 63, 2420-2427. https://doi.org/10.1109/TCOMM.2015.2441691

[4] Padovani, R. and Wolf, J. (1986) Coded Phase/Frequency Modulation. IEEE Transactions on Communications, 34, 446-453. https://doi.org/10.1109/TCOM.1986.1096564

[5] Periyalwar, S.S. and Fleisher, S.M. (1992) Multiple Trellis Coded Frequency and Phase Modulation. IEEE Transactions on Communications, 40, 1038-1046. https://doi.org/10.1109/26.142794

[6] Ugrelidze, N., Shavgulidze S. and Sordia, M. (2019) New Generalized Multistream Spatial Modulation for Wireless Communications. 2019 Wireless Days (WD), Manchester, 24-26 April 2019, 1-7. https://doi.org/10.1109/WD.2019.8734195

[7] Ugrelidze, N., Shavgulidze, S. and Sordia, M. (2020) New Four-Dimensional Signal Constellations Construction. IET Communications, 14, 1554-1559. https://doi.org/10.1049/iet-com.2019.0528

[8] Thomas, C., Weidner, M. and Durrani, S. (1974) Digital Amplitude-Phase Keying with M-Ary Alphabets. IEEE Transactions on Communications, 22, 168-180. https://doi.org/10.1109/TCOM.1974.1092165 
[9] Freudenberger, J. and Shavgulidze, S. (2017) Signal Constellations Based on Eisenstein Integers for Generalized Spatial Modulation. IEEE Communications Letters, 21, 556-559. https://doi.org/10.1109/LCOMM.2016.2630701

[10] Freudenberger, J., Rohweder, D. and Shavgulidze, S. (2018) Generalized Multistream Spatial Modulation With Signal Constellations Based on Hurwitz Integers and Low Complexity Detection. IEEE Wireless Communications Letters, 7, 412-415. https://doi.org/10.1109/LWC.2017.2780092 